\title{
New method to improve the accuracy of quench position measurement on a superconducting cavity by a second sound method
}

\author{
ZhenChao Liu, ${ }^{1}$ Michael Kelly, ${ }^{2}$ and Alireza Nassiri ${ }^{2}$ \\ ${ }^{1}$ Institute of High Energy Physics, Chinese Academy of Sciences, Beijing 100049, China \\ ${ }^{2}$ Argonne National Laboratory, Argonne, Illinois 60439, USA \\ (Received 7 March 2012; published 19 September 2012)
}

\begin{abstract}
Quench is a common phenomenon in a superconducting cavity and often limits the accelerating gradient of the cavity. Accurate location of the quench site, typically located at a material or geometrical defect, is the key to improve the cavity accelerating gradient. Here, the second sound propagation in liquid helium II is used to detect the quench location on the cavity. The technique is relatively convenient and complements the traditional temperature mapping which measures the "prequench" temperature rise on the cavity using an array of sensors. The speed of the second sound in liquid helium II is roughly $1.7 \mathrm{~cm} / \mathrm{ms}$ at $2 \mathrm{~K}$ which is sufficiently fast to provide a millimeter-size position resolution. However, the dynamics of the quench at the cavity surface are also found to significantly affect the achievable resolution with real cavities. Here we use a dynamic quench model, based on ANSYS, to calculate the quench area and the temperature distribution on the cavity. The detection error caused by the thermal conduction in the niobium was calculated.
\end{abstract}

DOI: 10.1103/PhysRevSTAB.15.092001

PACS numbers: 29.20.Ej

\section{INTRODUCTION}

Cavity quench is a thermal-magnetic break down phenomenon in superconducting cavities. It is usually caused by the defects on or near the radiofrequency (rf) surface of the superconducting cavity. Quench also typically limits the maximum achievable surface magnetic field to a value less than the thermodynamic critical field $(\sim 200 \mathrm{mT}$ $[1,2])$. Such defects have typical radius from tens to hundreds of microns and consist of various material and/ or geometrical anomalies.

The quench propagation is determined by normal state properties of the niobium, in particular, the resistivity and thermal conductivity. A defect will not initiate quench when the field gradient $H$ is sufficiently low. However, when the field increases, the power loss in the defect increases proportional to $H^{2}$, leading to heating of the surrounding area and eventually to a transition to the normal conducting state. If the area of the normal region exceeds a value, determined by a thermal balance relation, a quench is initiated. This is sometimes referred to as thermal runaway. Because of the proportionality between power loss and field gradient of squared $H$, the quench position often occurs near the region of maximum magnetic field.

When liquid helium is cooled below lambda point $(2.17 \mathrm{~K})$, the entropy flow in the liquid helium which is referred to as "second sound" can be used to locate the

Published by the American Physical Society under the terms of the Creative Commons Attribution 3.0 License. Further distribution of this work must maintain attribution to the author(s) and the published article's title, journal citation, and DOI. position of a quench on cavities working below $2.17 \mathrm{~K}$ [3]. The quench position in a multidimensional system can be derived from the time of flight from an array of detectors or from a single movable detector.

Figure 1 shows schematically how the distance is measured using second sound. The intrinsic response time of

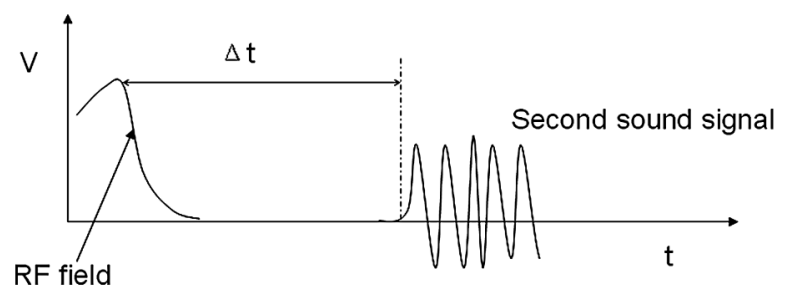

FIG. 1. Measurement of quench point by second sound.

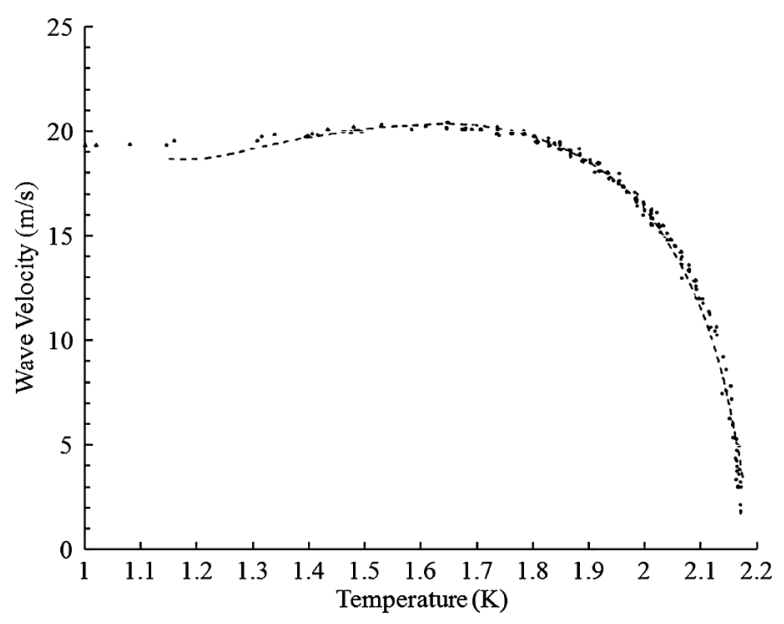

FIG. 2. Second sound velocity versus temperature. 


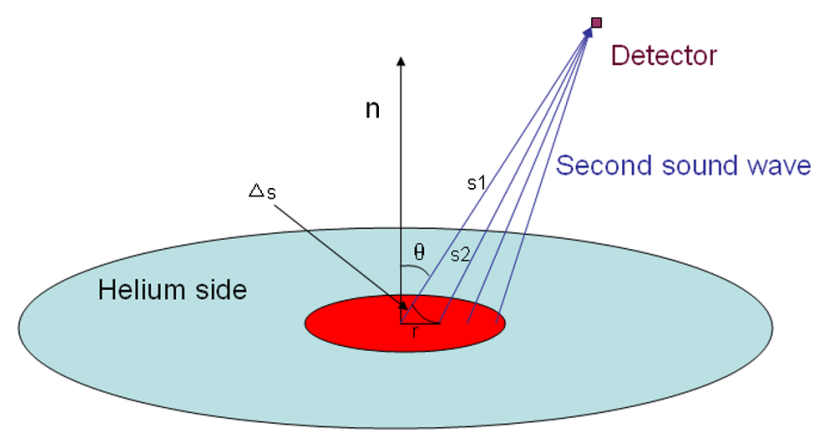

FIG. 3. Second sound signal from different position of niobium sheet.

the second sound thermometer is fast, such as a germanium resistor, typically 200 microseconds, so that an accurate distance can be determined by measuring the time interval from the initial drop in the $\mathrm{rf}$ field to the first rise of the second sound signal. The distance can be calculated by the measured time interval and the second sound velocity. Figure 2 shows the second sound velocity in He II [4]. However, the second sound signal is the result of heat deposited into an extended area on the cavity that grows with time as shown in Fig. 3. Therefore how to determine the source position from second sound signal is the key to improve the accuracy of measurement.

\section{ANALYSIS MODEL}

\section{A. Quench model}

We have used a dynamic quench model for cavity quench simulation. We simplify the model by assuming the defect is at the position of maximum magnetic field. Furthermore, we ignore the rf losses in the superconducting region (BCS resistance and residual resistance) as they are small compared with the normal state losses. This assumption will later be verified by comparison with experimental results.

A half wave cavity developed for the heavy ion acceleration at Argonne National Laboratory (ANL) is used as an example and some quench data have been obtained in early experiments. Table I shows some relevant parameters of the half wave cavity.

Figure 4 shows the model used in ANSYS [5]. The defect is on the center of the niobium surface. The magnetic field is assumed to be uniformly distributed over the quench area. The outside of the niobium is in contact with a $2 \mathrm{~K}$ helium bath and the thickness of the niobium sheet is $3 \mathrm{~mm}$, as for the half wave cavity. The total radius of the niobium sheet is $16 \mathrm{~cm}$ for this model which is larger than the radius of the region over which there is significant temperature rise during the quench. Cavities with substantially larger stored energy may require simulation over a larger niobium surface.
TABLE I. Parameters of the ANL half wave cavity.

Parameters at $1 \mathrm{MV} / \mathrm{m}$

Frequency

$U$

$172.5 \mathrm{MHz}$

$B_{p k}$

$0.345 \mathrm{~J}$

$E_{p k}$

$7.8 \mathrm{mT}$

Thickness of niobium

$2.9 \mathrm{MV} / \mathrm{m}$

RRR

$3 \mathrm{~mm}$

He bath temperature
$2 \mathrm{~K}$

\section{B. Normal region}

The transition temperature for niobium decreases with magnetic field on the superconducting niobium surface [6]. This temperature is given by

$$
T_{c}=9.2\left(1-\frac{B}{200}\right)^{1 / 2} .
$$

Here $B$ is the magnetic field in units of $\mathrm{mT}$.

The power dissipation is given by

$$
\dot{Q}=\frac{1}{2} \int R_{s}|H|^{2} d s .
$$

$H$ is the magnetic field, and $R_{S}$ is the surface resistance of normal state niobium.

We assume $H$ is constant, so that the unit area power dissipation is

$$
P=\frac{1}{2} R_{s}|H|^{2} .
$$

Finally, the normal region rf surface resistance is given by

$$
R_{s}=\sqrt{\frac{\omega \mu}{2 \sigma}},
$$

where $\omega=2 \pi f, f$ is the rf frequency, $\mu$ is the permeability of niobium, and $\sigma$ is the electrical conductivity of niobium. Figure 5 shows the normal state surface resistance used for the model. Surface resistance of $10 \mathrm{~m} \Omega$ is adopted for the defect region used in the simulation.

\section{Stored energy}

The stored energy in the cavity and the forward power of the input coupler is available in simulation in this model. The power in the cavity is

$$
U=U_{0}-\Delta Q+P_{f} \Delta t .
$$

Here, $U_{0}$ is the stored energy in the cavity before quench, $\Delta Q$ is the energy dissipated on the normal region and defect, $P_{f}$ is the forward power into the cavity, and $\Delta t$ is the time step.

\section{Boundary condition}

There are two types of heat transfer from the niobium surface to helium II (He II) in this model. The Kapitza conductance is used for the low heat flux transfer simulation while the heat flux is less than $1 \mathrm{~W} / \mathrm{cm}^{2}$ from niobium 


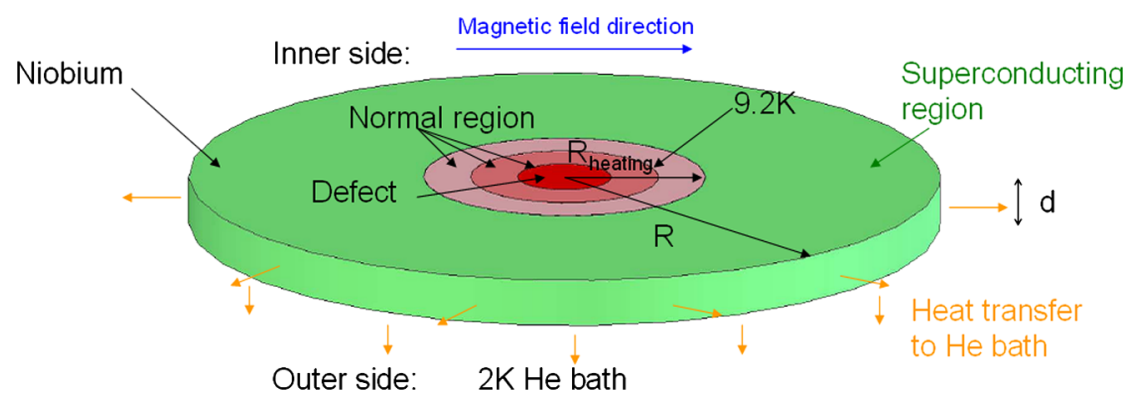

FIG. 4. Dynamic model for cavity quench simulation.

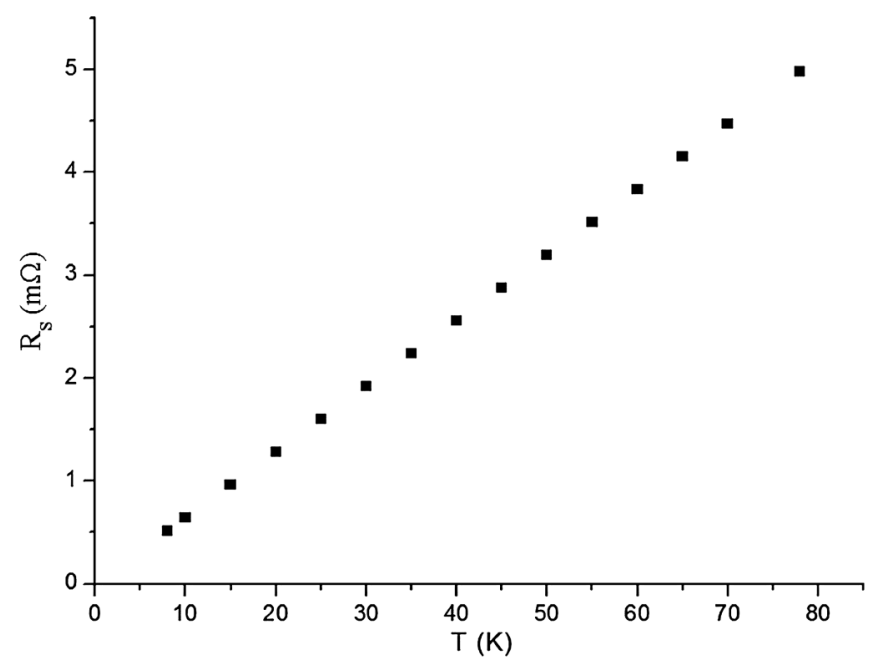

FIG. 5. $172.5 \mathrm{MHz}$ half wave cavity normal state region surface resistance $(\mathrm{RRR}=250)$.

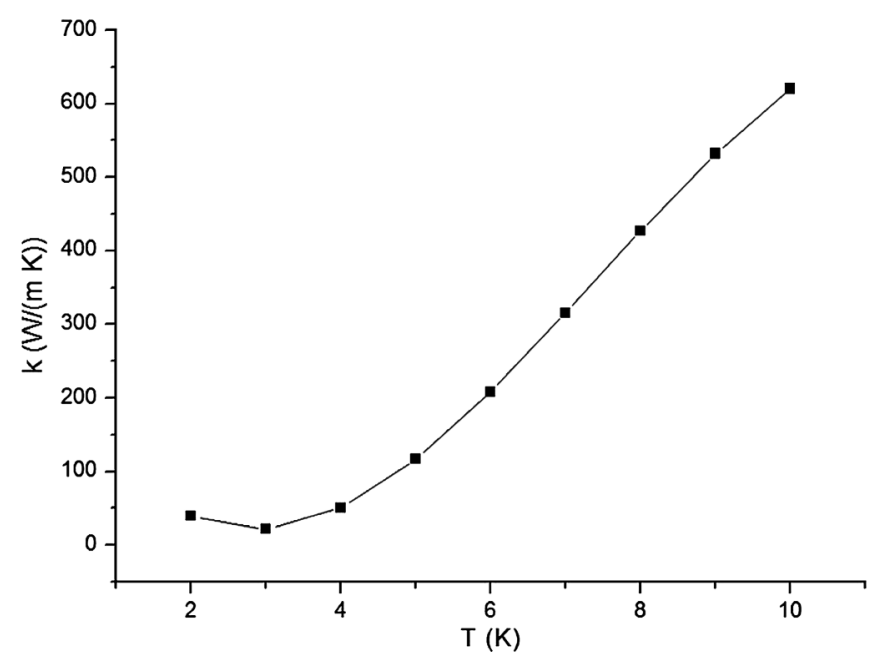

FIG. 6. Thermal conductivity of niobium $(\mathrm{RRR}=250)$.

surface to He II. The formula of Kapitza conductance [6] for $2 \mathrm{~K}$ helium bath is adopted for the low heat flux transfer. Film boiling will happen when the heat flux between the niobium surface and He II exceeds the critical heat flux for $\mathrm{He}$ II of $\sim 1 \mathrm{~W} / \mathrm{cm}^{2}$. Although there is a temperature

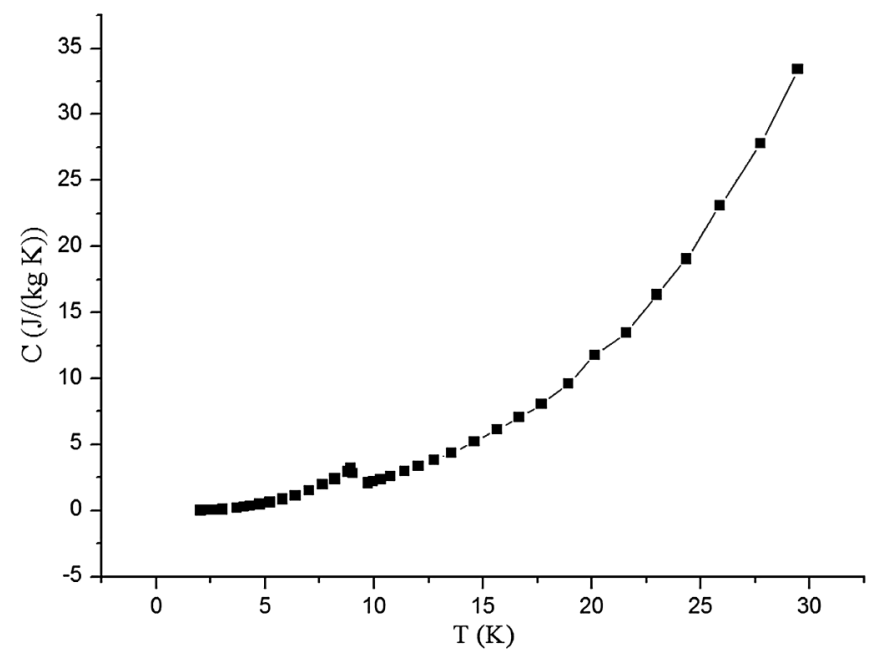

FIG. 7. Capacity of niobium.

discontinuity when heat flux exceeds the critical heat flux of He II, we make a simplification here and use the heat transfer coefficient for the flat surface [7] for the whole film boiling region.

\section{E. Properties of niobium}

Figure 6 shows the thermal conductivity of niobium of $R R R=250$. Linear interpolation is used for temperature from 10 to $300 \mathrm{~K}$. Figure 7 shows the specific heat of niobium [8]. Linear interpolation is used for temperature from 30 to $300 \mathrm{~K}$.

\section{ANALYSIS RESULTS}

\section{A. Quench results}

A cavity quench at $E_{\text {acc }}=10 \mathrm{MV} / \mathrm{m}$ for the half wave cavity has been simulated. Figure 8 shows the stored energy evolution in the cavity. The simulation shows that the normal region expands to a radius of $7 \mathrm{~cm}$ in $1 \mathrm{~ms}$ and that area remains in the normal state for about $160 \mathrm{~ms}$. On the niobium surface in contact with the helium there is film boiling in the quench area to a radius of $10 \mathrm{~cm}$ lasting for about $150 \mathrm{~ms}$. Clearly, the process of heat transfer from niobium surface to helium is much slower than the time 


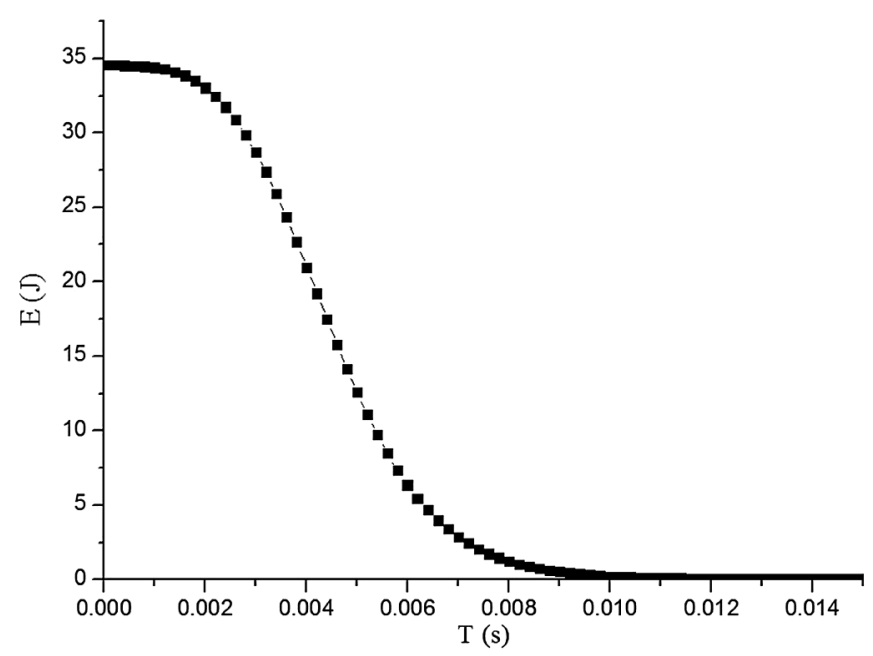

FIG. 8. Stored energy profile in the half-wave cavity.

scale of the decay of the field energy. Figure 9 shows the temperature distribution of the niobium surface contacting to helium.

\section{B. Comparison with experiment results}

In the quench experiment on the half wave cavity, a rectangular rf power pulse fed into the cavity and induced quench. The rf drive pulse was turned off several milliseconds after the quench was initiated. The same pulse structure is adopted in the simulation model. As the voltage in the cavity is proportional to the magnetic field, we compared the decay times of the voltage in the experiment and the magnetic field in simulation. The simulated decay time of $2.003 \mathrm{~ms}$ is in excellent agreement with the experimental value of $2.075 \mathrm{~ms}$. Figure 10 shows the voltage evolution of real quench and the magnetic field evolution of simulation.

\section{Influence on quench point location by second sound}

The second sound wave in the He II bath is caused by the sudden temperature rise on niobium surface. The second

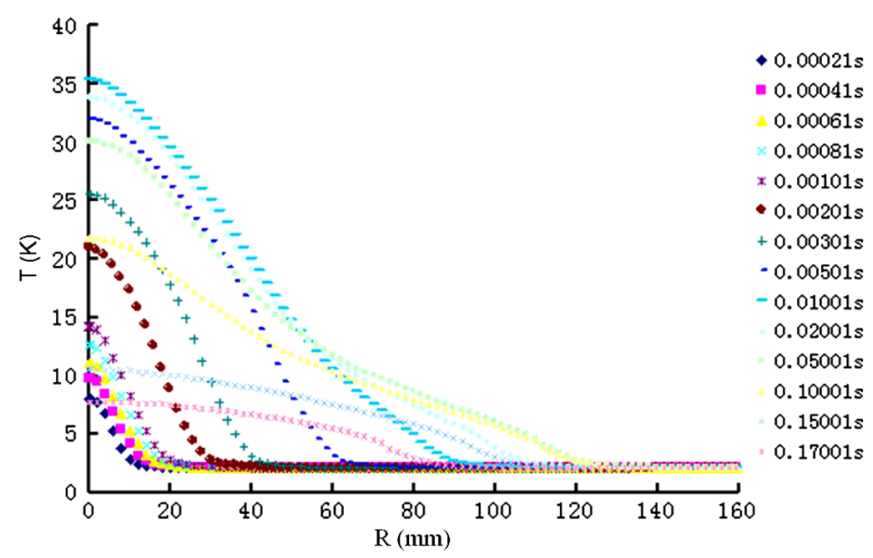

FIG. 9. Niobium surface temperature on the liquid helium side.
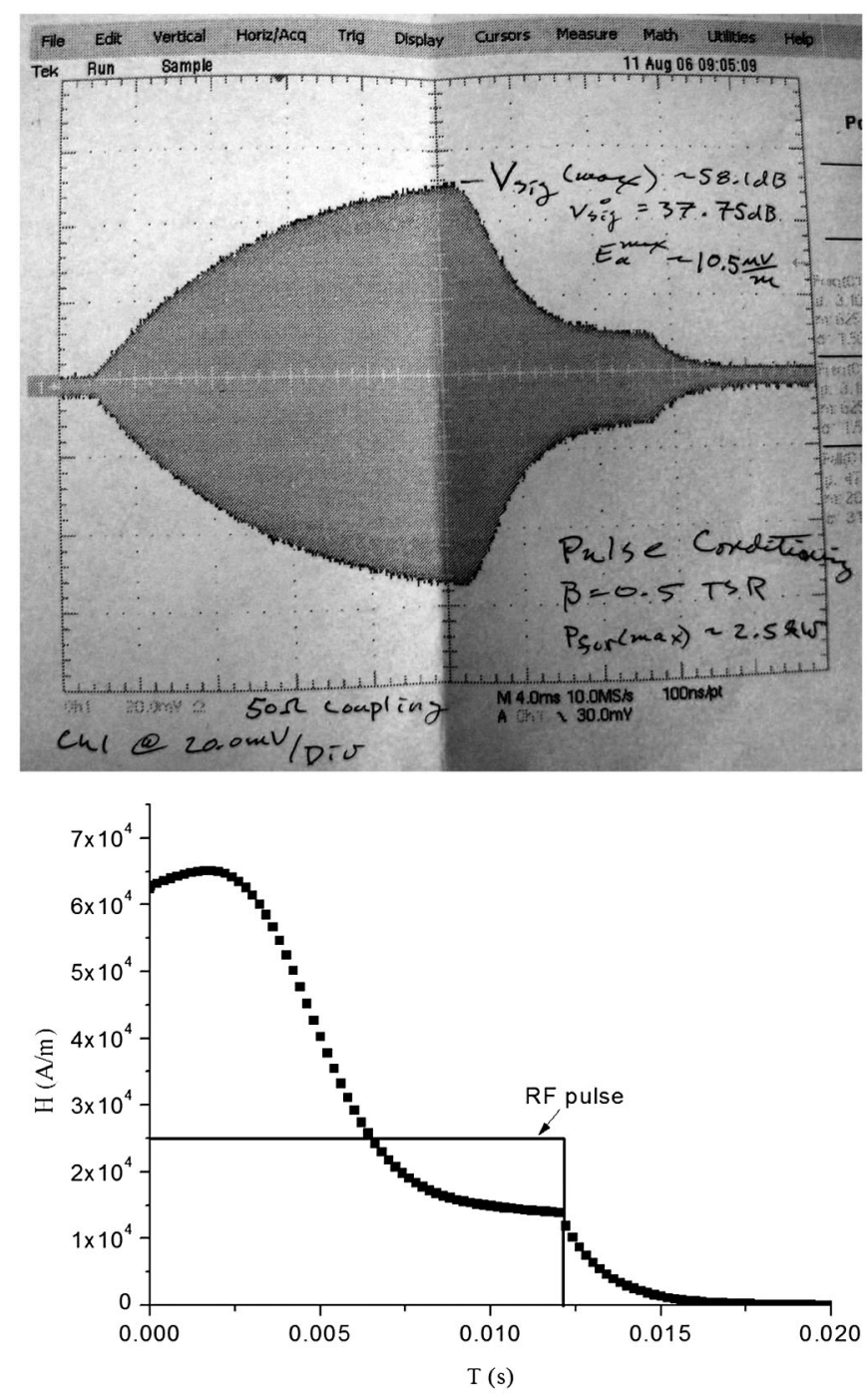

FIG. 10. Voltage evolution of real quench (top) and magnetic field evolution of simulation (bottom).

sound signal amplitudes shown on oscillator are influenced by the sensitivity of the detector, the distance between the source and the detector, the background noise in the helium bath, etc. Therefore only a sudden temperature rise (e.g., several hundreds milli-Kelvin) can cause the second sound signal on the oscillator. This was observed on the experiment of second sound caused by the thermoresistor. Figure 11 shows temperature distribution on the helium side niobium surface and the radius's evolution of certain temperatures during quenching.

The sudden temperature rise will emerge on the niobium surface around the initial quench point when the quench starts. Therefore the second sound wave caused by sudden temperature rise of the edge area may reach the detector first for some detection angles. The reason is that the second sound speed is not fast enough. Figure 12 shows the second sound signal's traveling in the helium. The second sound signal shown on the oscillator may be the 


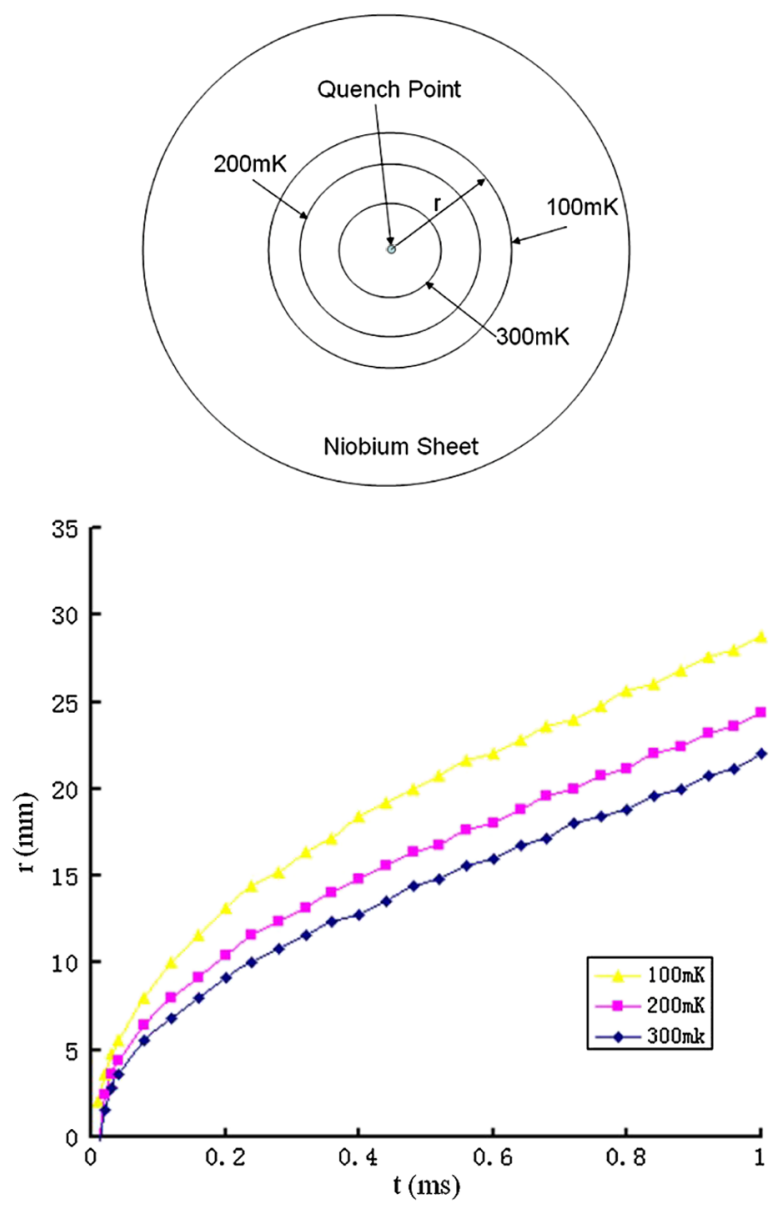

FIG. 11. Temperature rise evolution on the helium side niobium surface of quench area. Top: the sketch temperature distribution while quenching; bottom: the radius's evolution of certain temperature while quenching.

second sound wave $b$ caused by quench edge area. However, the distance between the detector and the initial quench point is calculated by the traveling time of second sound wave $a$. This time error should be compensated by calculation with the quench simulation results. For example, Fig. 13 shows the time errors calculated from the temperature distribution results of Fig. 9 with detection

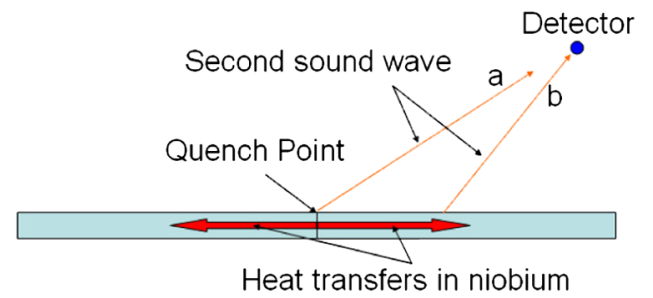

FIG. 12. Second sound wave propagation after quench happens. As the speed of heat transfer in niobium is fast, the temperature at the region near the defect can rise to a high value in a few hundred microseconds. This will cause the result of second sound wave $b$ (not from quench point) reaching the detector before second sound wave $a$ (from quench point).
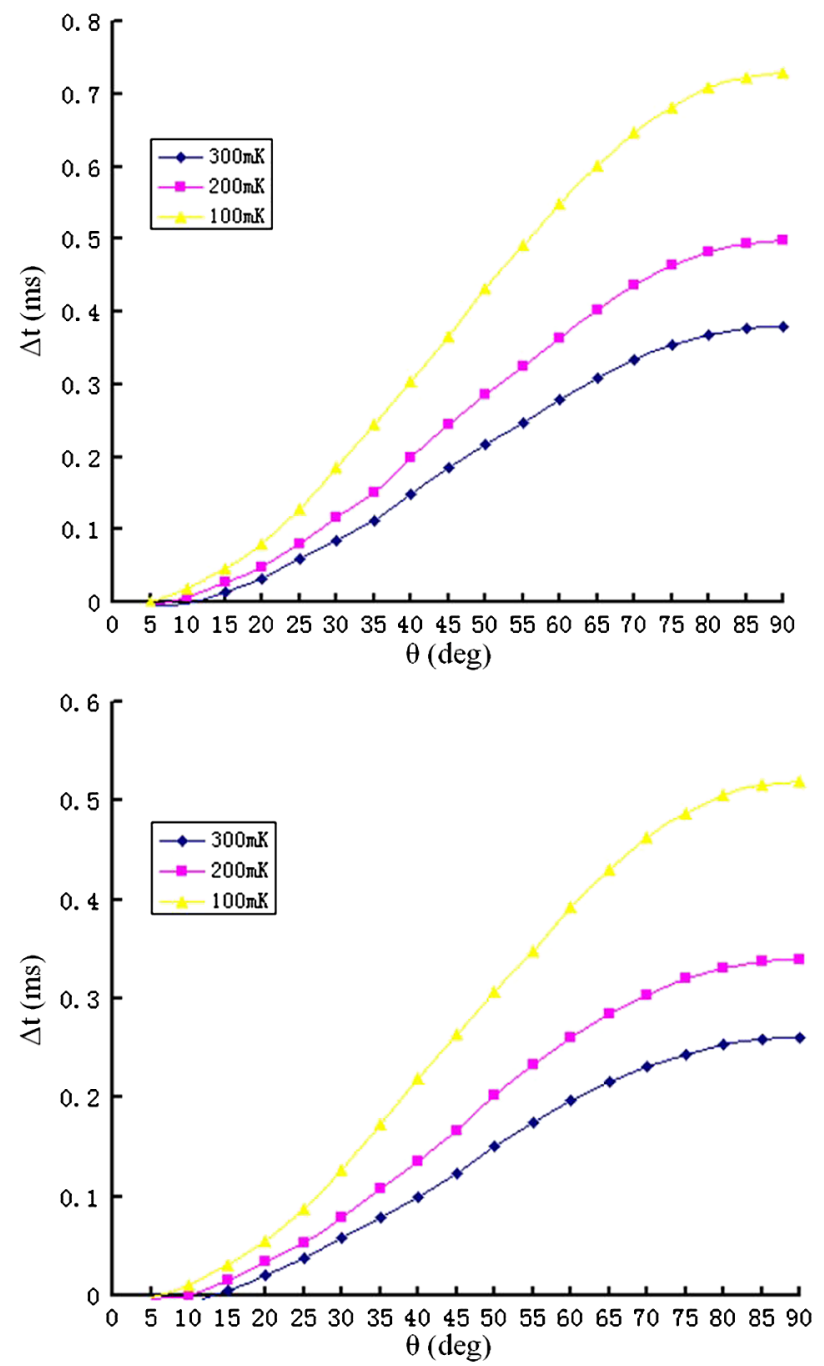

FIG. 13. Time error calculated for $50 \mathrm{~cm}$ away detection at different angles and system resolutions. $\theta$ is defined as in Fig. 3. Top: Second sound velocity at $2 \mathrm{~K}$ is adopted. Bottom: Second sound velocity at $1.8 \mathrm{~K}$ is adopted.

system resolutions of 100,200 , and $300 \mathrm{mK}$. The time errors are large for angles larger than about 40 degrees. For example, the time error is $0.42 \mathrm{~ms}$ when the detector is set 50 degrees with the niobium surface and $50 \mathrm{~cm}$ away from quench point with a system resolution of $100 \mathrm{mK}$ in $2 \mathrm{~K}$ helium bath. This is a large error comparing with the resolution of second sound detection $(\sim 3 \mathrm{~mm})$. The second sound velocity is about $2 \mathrm{~cm} / \mathrm{ms}$ in the $1.8 \mathrm{~K}$ helium bath. It is faster than the second sound velocity in the $2 \mathrm{~K}$ helium bath. Thus, the time error in the $1.8 \mathrm{~K}$ helium bath is smaller than in the $2 \mathrm{~K}$ helium bath for the detectors at the same position. This is also shown in Fig. 13.

As the temperature rise distribution on the helium side of the niobium surface is influenced by the stored energy in the cavity while quenching and the thermal conduction of niobium, the time errors will change with the quench condition and the niobium property (RRR). The time error 
will also change with the distance between the initial quench point and the detector.

\section{CONCLUSION}

A dynamic superconducting cavity quench model using ANSYS has been presented. Simulations were used to determine dynamic temperature distribution of the quench area. Simulation results indicate that the second sound detection may have errors as the thermal conduction in niobium is faster than the second sound speed. The paper provides a method to compensate the detection errors.

\section{ACKNOWLEDGMENTS}

The work of ZhenChao Liu was supported by the China Scholarship Council. Most of the work was done when ZhenChao Liu was working at ANL and PKU.
[1] H. R. Kerchner, D. K. Christen, and S.T. Sekula, Phys. Rev. B 24, 1200 (1981).

[2] J. Daams and J. P. Carbotte, J. Low Temp. Phys. 40, 135 (1980).

[3] Z. A. Conway et al., Oscillating Superleak Transducers for Quench Detection in Superconducting ILC Cavities Cooled with HE-II, in Proceedings of LINAC08, Victoria, BC, Canada, THP036.

[4] J. R. Pellam, Phys. Rev. 75, 1183 (1949).

[5] http://www.ANSYS.com.

[6] A. Aizaz and T.L. Grimm, Thermal Limitations in Superconducting RF Cavities: Improved Heat transfer at Niobium-Helium Interface, C2-O-06 at the 2005 CEC/ICMC Key Stone, Colorado, Advances in Cryogenic Engineering.

[7] J. G. Weisend II, Handbook of Cryogenic Engineering (Taylor \& Francis, London, 1998), p. 463.

[8] Chien Chou, David White, and H. L. Johnston, Phys. Rev. 109, 788 (1958). 